\title{
Agradecimento aos Revisores da Acta Médica Portuguesa
}

\section{Thank You, Reviewers of Acta Médica Portuguesa}

Miguel GUIMARÃES ${ }^{1,2,3}$, Tiago VILLANUEVA $\triangle^{4,5}$

Acta Med Port 2021 Feb;34(2):75-79 - https://doi.org/10.20344/amp.15747

Entre 1 de janeiro e 31 de dezembro de 2020, a Acta Médica Portuguesa recebeu 1625 submissões através da sua plataforma electrónica de gestão do processo editorial (Open Journal System). Destes artigos, 217 foram publicados ao longo das 11 edições regulares. No mesmo período, e contando com uma base de dados com 6807 revisores no final do ano, concluíram-se 775 processos de revisão por pares.

Decidir quais os trabalhos a publicar, procurando constituir uma mais-valia efectiva para os nossos leitores e assim contribuir para a inovação da investigação e da prática clínica, e para a divulgação do conceito da moderna autoria científica, é um processo complexo. Neste âmbito, a colaboração dos peritos a quem solicitámos a avaliação dos trabalhos propostos para publicação é fundamental.

A Acta Médica Portuguesa é a única revista médica portuguesa indexada de âmbito generalista, com uma audiência de mais de 45000 médicos portugueses, outros profissionais de saúde, decisores e população em geral. Como tal, é imprescindível o contributo de especialistas das várias áreas, que nos apoiam na identificação dos temas de maior relevância, comentam a pertinência dos estudos propostos, realçam as linhas inovadoras das metodologias apresentadas, etc.

Gostaríamos de salientar a necessidade de termos mais revisores inscritos na plataforma, para que possamos diminuir os tempos de decisão das submissões. A inscrição na plataforma OJS pode ser feita rapidamente nesta página: https:// www.actamedicaportuguesa.com/revista/index.php/amp/user/register.

A todos e a cada um de vós - que de forma contínua demonstram reconhecer o peer-review como um dever de cidadania científica - se dirige o sincero agradecimento da Ordem dos Médicos e da Acta Médica Portuguesa.

Lisboa, 13 de Janeiro de 2021

\author{
Miguel Guimarães \\ Bastonário da Ordem dos Médicos \\ Tiago Villanueva \\ Editor-Chefe
}

A lista em baixo enumera os revisores que ao longo de 2020 procederam à avaliação de artigos a pedido da nossa publicação.

Revisores com seis avaliações concluídas:

Diogo Almeida

Maria Margarida Costa Ferreira Bandarra

Revisores com cinco avaliações concluídas:

José Luís Alves

Rui Afonso Cernadas

Vera Moniz-Pereira

\footnotetext{
1. Bastonário. Ordem dos Médicos. Lisboa. Portugal.

2. Co-Editor. Acta Médica Portuguesa. Lisboa. Portugal.

3. Serviço de Urologia. Hospital de São João. Porto. Portugal.

4. Editor-Chefe. Acta Médica Portuguesa. Lisboa. Portugal.

5. Médico de Família. Unidade de Saúde Familiar Reynaldo dos Santos. Póvoa de Santa Iria. Portugal.

$\triangle$ Autor correspondente: Tiago Villanueva. tiago.villanueva@ordemdosmedicos.pt

Recebido: 13 de janeiro de 2021 - Aceite: 13 de janeiro de 2021 - Online issue published: 01 de fevereiro de 2021

Copyright (C) Ordem dos Médicos 2021
} 
Revisores com quatro avaliações concluídas:

Airlane Pereira Alencar

Maria Helena Almeida

Eleonora Paixão

Francisco Caramelo

Isabel De Santiago

Micaela Antunes

Nuno Alegrete

Patrícia Almeida

Revisores com três avaliações concluídas:

Ana Carina Ferreira

Ana Luísa Almeida

Antónia Campos

António Jorge Cabral

Bernardo Gomes

Cecília Castro

Fausto Carvalheira

Filomena Carnide

Francisco Cunha

Guiomar Gonçalves Oliveira

Helena Maria Carvalho

Hugo Sousa Câmara
Inês Brás Marques

Isabel Boaventura

Isabel Vitória Figueiredo

J.M. Azevedo-Pereira

João Sollari Lopes

Leonor Pinto Amaro

Luís Sousa

Luiz Miguel Santiago

Maria Clara Bicho

Maria João Aleixo

Mariana Brandão

Nuno Madeira
Pedro Afonso

Pedro Aguiar

Pedro Escada

Revisores com duas avaliações concluídas:

Adelaide Costa

Álvaro Azevedo

Ana Calistru

Ana Cardoso

Ana Correia Campos

Ana Filipa Margalho

Ana Júlia Pedro

Ana Mafalda Reis

Ana Maria Abreu

Ana Nunes Barata

Ana R. Barbosa

Ana Rita Clara

Ana Rosa Costa

Ana Vieira Marques

André Peralta Santos

Andreia Gomes Costa

António Jorge Ferreira

Aurélio Maldonado

Bruno Damásio

Bruno Rocha

Carla Sofia Silva

Carlos Góis

Carolina Santos

Carolina Vidal

Catarina Pulido

Celeste Dias

Cláudia Camila Dias

Cláudia Costa

Constança Penedos

Cristina Carvalho Gouveia

Cristina Maria Santos

Dalila Veiga

Daniel Barrocas

Daniel Machado

Daniela Cunha

Davide Carvalho
Dina Gaspar

Dina Salvador

Dinarte Nuno Viveiros

Duarte Pedro Tavares

Duarte Vital Brito

Edgar Mesquita

Eduardo Breda

Eduardo Cruz

Eduardo Carqueja

Eduardo Palha-Fernandes

Edward Araújo Júnior

Elisabete Ramos

Eugénia Anes

Fábio Cota Medeiros

Fátima Falcão

Fátima Serrano

Felipe Andrade

Fernando Almeida

Fernando Trancoso Vaz

Filipa Novais

Filipe Caseiro Alves

Filipe Palavra

Francisco Antunes

Giovani Loióla Silva

Gonçalo Melo

Henrique Alexandrino

Hercília Guimarães

Hernâni Pombas Caniço

Hugo Rocha

Ilda Massano Cardoso

Inês Correia-Sá

Inês Palma Reis

Inês Sequeira

Isabel Antunes

Isabel José de Sousa

Isabel Natário
Paulo Santos

Pedro Castro

Pedro Luís Morgado

Pedro M. A. R. Correia

Ricardo Soares dos Reis

Rita Marques

Rosa Amélia Dantas

Sofia Martins Amante

Tiago Taveira-Gomes

Vasco Ricoca Peixoto

Zaida Aguiar Sá Azeredo
J. E. Passos Coelho

J. Torres da Costa

J.M. Caldas Almeida

Joana Almeida

Joana Alves

Joana Carreteiro

Joana Urzal

João Anselmo

João Borges Costa

João Gama Marques

João Leonel Felgueiras

João Nuno Ramos

João Paulo Branco

José Aguiar

José António Mariz

José Manuel Calheiros

José Pedro Boléo-Tomé

José Pereira

Luís Albuquerque

Luís Guilherme Casimiro

Luísa Alves

Luísa Vilalonga Pereira

Mafalda Felgueiras

Manuel Bragança Pereira

Manuel Caneira

Manuel Luís V. Capelas

Marco Costa

Margarida Gonçalo

Maria Goreti Catorze

Maria Helena Pimentel

Maria João Costa Nunes Lobão

Maria João Polidoro

Maria José Sá

Maria Leonor Fernandes

Maria Raquel Carvalho

Maria Teresa Herdeiro 
Maria Vieira

Mariana Carlos Alves

Mário Fontes e Sousa

Marlene Saraiva

Marta Azenha

Meireles Brandão

Mesquita Bastos

Miguel Gouveia

Miguel Ricou

Nuno Abecasis

Patricia S. Carrilho

Patrick Alves

Paula Milheiro-Oliveira
Paula Pinto de Freitas

Paula Rosa de Aldomiro

Paulo Jorge Nogueira

Paulo Martins

Pedro Brandão

Pedro Oliveira

Pedro Simões

Ricardo Almendra

Roberta Frontini

Rosália Sá

Rui Campos

Rui Moreno
Sandra Saraiva Ferreira

Sérgio Chacim

Stéfanie Pereira

Teresa Almeida Santos

Teresa Almodovar

Teresa Amaral

Tiago Alfaro

Tiago Torres

Úrsula Segura

Vera Afreixo

Victor Bezerra

Zélia Anastácio

Revisores com uma avaliação concluída:

Adelina Costin

Adriana Belo Cabete

Adriana Coelho

Agostinho Moreira de Sousa

Alexandra Bayão Horta

Alexandra Seabra Dinis

Alexandre Castro Caldas

Alexandre Morais Nunes

Aline Campos Paiva

Amélia Almeida

Amélia Moreira

Amélia Tavares

Amílcar Oliveira

Ana Carocha

Ana Cortesão Costa

Ana Cristina Moreira

Ana Escoval

Ana Gameiro

Ana Luísa Ribeirinho

Ana Mafalda Carvalheiro

Ana Maria Stamm

Ana Oliveira

Ana Paula Duarte

Ana Paula Harfouche

Ana Raquel Rodrigues

Ana Rita Prior

Ana Sofia Correia

Ana Sofia Pinto

Ana Sousa Menezes

Ana Xavier

Anabela Afonso

Anabela Barcelos

Anabela Pinto

Anderson Garcez

André Amaral

André Cerejeira

André Carrão

André M. Graça

André Weigert

Andrea Sofia Dias

Andreia Leite

Andreia Sofia Costa Teixeira
Andreia Sofia Veiga

António Andrade

António Assunção

António Barbosa

António Cardoso Fernandes

António Coimbra Matos

António Faria Vaz

António J. Atalaia

António Lourenço Marques

António Silva Graça

António Tralhão

Aristides Machado-Rodrigues

Armando Flores-Rebollar

Arminda Manuela Gonçalves

Bárbara Cesar Machado

Bárbara Marques

Bárbara Oliveiros

Bernardo Pereira

Bruno Heleno

Camila Franco

Cândida Cancelinha

Carla André

Carla Nunes

Carla Santos

Carlos Carvalho

Carlos Pontinha

Carlota Quintal

Carolina Lemos

Carolina Vaz de Macedo

Catarina Duarte Santos

Catarina Freitas

Catarina Prior

Catarina S. Nunes

Cátia Azenha

Clara Chamadoira

Cláudia Borbinha

Cláudia Pavão Matos

Constantino Sakellarides

Cristina Amaro

Cristina M. Jorge

Cristina Padez

Cristina Ribeiro
Cristina Teixeira

Daniel Pinto

Daniel Virella

Daniela Carvalho

Dário Ferreira

David Peres

Débora Miranda

Diana Campos Lopes

Diana Penha

Diana Póvoas

Diogo Cerejeira

Doug McWhinnie

Duarte da Silva Soares

Duarte Nuno Vieira

Dulce Oliveira Gomes

Edgar Tavares-da-Silva

Edson Oliveira

Elena Atienza-Macias

Élia Baeta

Elisa Pedro

Elizabete Neutel

Elsa Landim

Eurico Castro Alves

Fernando Gomes

Fernando Abelha

Filipa Lança

Filipa Machado Vaz

Filipa Quaresma

Filipa Sofia Silva

Filipe Antunes

Filipe Coutinho

Filipe Froes

Filomena Azevedo

Filomena Melo

Francisco Gonçalves

Gabriel Anacleto

Gisele Barbosa

Glória Cunha Velho

Gonçalo Cruz Marau

Goreti Lobarinhas

Graça Oliveira

Graciete Bragança 
Gracinda Guerreiro

Guilherme Amaral Mendes

Guilherme Ferreira-Dos-Santos

Guilherme Gonçalves

Guilhermina Rego

Gustavo Tato Borges

Harith Al-Warid

Hélder Filipe

Hélder Ribeiro Pereira

Henrique Barros

Henrique Duarte

Henrique Messias

Hugo de Barros Viegas

Inês Balacó

Inês Sanches

Isabel Fonseca

Isabel Galriça Neto

Isabel Sampaio

Isabel Saraiva de Melo

Isabel Silva

Ivo Álvares Furtado

Ivo Dinis Oliveira

Ivone Duarte

Jaime Nina

Jessica Lomba

Joana Alves Vaz

Joana Arcângelo

Joana Bastos

Joana Belo

Joana Revés

Joana Cabete

Joana Canais

Joana Couceiro

Joana Lopes

Joana Moreira Barros

Joana Moura Ferreira

Joana Nogueira

Joana Santos Costa

João Gonçalves

João Aranha

João Beato

João Breda

João C. Pimentel

João Cabo

João Cerqueira

João Gonçalves Pereira

João Massano

João Melo Alves

João Páscoa Pinheiro

João Pinheiro Amorim

João Sargento-Freitas

Joaquim Andrade-Gomes

Joaquim Teixeira

Joaquim Varandas

Jorge Barbosa

Jorge Costa Santos

José Alberto Pereira
José Augusto Rodrigues Simões

José Carlos Campos

José Daniel Rodrigues

José Luís Metello

José Manuel Boavida

Juan Javier Gervas

Júlio Castro Soares

Lara Queirós

Leandro Almeida

Leandro Silva

Lélita Santos

Leonor Boto

Lia Rodrigues e Rodrigues

Lilia Aparecida Kanan

Luciana Ricca

Luís Agualusa

Luís Antunes

Luis Carreto

Luís Mendes Pedro

Luís Miguel Monteiro

Luís Morais

Luís Nobre Pereira

Luís Pisco

Luís Ruano

Luís Silva

Luís Silveira

Luiz Pinto

Madalina Guz

Manuel Cunha

Manuel Pizarro

Manuel Rosete

Manuela Carvalheiro

Manuela Oliveira

Margarida Castel-Branco

Margarida Figueiredo Dias

Margarida Fonseca Cardoso

Margarida Maria Marques

Margarida Silvestre

Maria Alexandra Mineiro

Maria da Piedade Moreira Brandão

Maria do Céu Machado

Maria do Rosário Martins

Maria Gabriela Andrade

Maria Inês Sequeira

Maria João Simões

Maria José Costeira

Maria Paula Faria

Mariana Amaral

Mariana Barosa

Mariana Correia

Mariana Donato

Mariana Morgado

Mariana Sousa Batista

Marina Carvalho

Marina Pinheiro

Mário Rodrigues Neto

Marta Alves
Marta Alves

Marta Pingarilho

Michał Zarobkiewicz

Miguel Bigotte Vieira

Miguel Gago

Miguel Gouveia

Miguel Grunho

Miguel Julião

Miguel Leão

Miguel Palha

Miguel Mendes

Miguel Soares-Oliveira

Miguel Salgado

Miguel Vieira

Miguel Abreu

Milena Paneque

Mónica Caldeira

Mourão Carvalho

Natacha Amaral

Natalia Marto

Nelson Gilberto

Noémia Afonso

Nuno Moreira Fonseca

Nuno Trovão

Óscar Ferraz Camacho

Pablo Santos

Patrícia Dinis Dias

Paula Borralho

Paula Bruno

Paula Neves

Paulo Barreto

Paulo Paixão

Pedro Alves

Pedro Coelho

Pedro Coelho Barata

Pedro Gaspar da Costa

Pedro Abreu

Pedro Serrano

Rafaela Ponte Sousa

Raquel Braga

Raquel Marques

Raquel Vieira

Regina Caldas

Ricardo Dinis-Oliveira

Ricardo Bacchi

Rita Gaio

Rita Gameiro

Rita Sá Machado

Rui Barranha

Rui Branco

Rui Vasconcellos Guimarães

Rui Mateus Marques

Rui Pombal

Rute Martins

Sara Drumond Freitas

Sara Magalhães

Sérgio Campainha 
Sofia Nunes Oliveira

Sofia R.T. Nunes

Sónia Duarte Oliveira

Sónia Freitas
Susana Teixeira Sousa

Teresa Bandeira

Teresa Cardoso

Tiago Fonseca
Tiago Marques

Tomás Teodoro

Valeriano Leite

Vera Trocado 